\title{
Article \\ Genetic Features of Multi Drug Resistant (MDR) Klebsiella pneumoniae and its Plasmids
}

\author{
Begoña Fuster ${ }^{1, *}$, Nuria Tormo ${ }^{1}$, Carme Salvador ${ }^{1}$, Neris García ${ }^{3}$, Fernando González-Candelas ${ }^{3}$ \\ and Concepción Gimeno ${ }^{1,2}$ \\ 1 Microbiology Department, Valencia General University Hospital (SPAIN) \\ 2 Faculty of Medicine, University of Valencia \\ 3 FISABIO, Valencia \\ * Correspondence: begona.fuster@gmail.com; Tel.: +34 627018034
}

\begin{abstract}
Klebsiella pneumoniae, a major cause of both hospital and community-acquired infections, is listed by the World Health Organization as a critical priority antibiotic-resistant bacterial pathogen. With the appearance of sequencing techniques such as Next-generation Sequencing (NGS), there is the possibility to obtain the whole genome of the bacteria, getting to know all antimicrobial resistance determinants. The purpose of this study has been to apply this new technology to clinical microbiology, in order to characterize the resistome present in carbapenemresistant K.pneumoniae strains isolated in a tertiary hospital in Valencia, Spain. A total of 234 isolates were prepared for whole-genome sequencing with Ilumina MiSeq, and sequences were later studied for antimicrobial resistance genes, sequence-typing and plasmids. Sequence-typing showed four major circulating clones in our hospital settings: ST11, ST307, ST101 and ST147, carrying different plasmids and different resistance determinants such as OXA-48 and NDM-1 carbapenemase. Application of new technologies such as whole-genome sequencing in clinical microbiology gives advantages when it comes to rapid therapy adjustment, consequently improving the patient's clinical outcomes.
\end{abstract}

Keywords: antimicrobial; resistance; plasmids; sequencing

\section{Introduction}

Klebsiella pneumoniae, a major cause of both hospital and community-acquired infections, is listed by the World Health Organization as a critical priority antibiotic-resistant bacterial pathogen [1]. The dramatic increase in prevalence of infections caused by these multidrug-resistant (MDR) and extremely drug resistant (XDR) microorganisms belonging to the Enterobacteriaceae group poses a great concern, since they are common natural inhabitants of our microbiome [2].

K.pneumoniae resistant to carbapenems poses the problem of antibiotic resistance, not only regarding ß-lactams but also other first-lane antibiotics such as fluorquinolones and aminoglycosides. This is usually due to the fact that many resistance genes are carried in the same mobile element and are transferred together from one isolate to another. In the daily laboratory routine at health-care centers only detection of $B$-lactams resistance genes is performed, both phenotypically and genotypically. This is because PCRs and detection kits are more easily available for detection of $\mathrm{B}-$ lactams resistance. However, detection of other resistance determinants such as aminoglycoside methylases or $Q n r$ genes providing resistance to fluorquinolones are not usually performed.

With the appearance of sequencing techniques such as Next-generation Sequencing (NGS), there is the possibility of obtaining the whole genome of the bacteria, getting to know all antimicrobial resistance determinants. This may help to know which resistance determinants are transferred together in the same plasmid or transposon, helping decide the final and adequate antimicrobial therapy for the patient. For this reason, the purpose of this study has been to apply NGS to clinical microbiology in order to characterize the resistome present in the carbapenem-resistant K.pneumoniae strains isolated in a tertiary hospital in Valencia, Spain. 


\section{Methods and Materials}

\subsection{Isolates Selection and Sequencing}

Out of the 300 isolates of Klebsiella pneumoniae resistant to carbapenems isolated, 234 were prepared for whole-genome sequencing (around 80\%). The remaining 66 of the 300 isolates were discarded due to a lack of growth, contamination of the culture plates, or having multiple phenotypes suggestive of contamination with different species.

All the 234 microorganisms were plated on blood agar (Beckton Dickinson) and incubated overnight at $37^{\circ} \mathrm{C}$. Genomic DNA extraction was performed in the automated system MagCore (RBD Science). Isolates were sequenced using the Illumina MiSeq Illumina (San Diego, CA, USA) platform with 300x2 "paired-ends".

\subsection{Analysis of Sequenced Data}

FastQC was used to evaluate quality and trim the Illumina sequence reads if necessary [3]. Sequence-typing, identification of resistance genes and plasmid analysis was performed using ARIBA and its functions: MLST-calling, Resfinder and Plasmidfinder, respectively.

\subsubsection{Identification of Sequence-types (STs).}

The seven-gene ST of all the isolates was determined using the multilocus sequence typing (MLST) [4] calling function of ARIBA v.2.6.1 [5] and the 'Klebsiella pneumoniae' database from PubMLST [4].

\subsubsection{Identification of carbapenemase genes.}

Carbapenemase genes (blaKPC-like, blaNDM-like, blaVIM-like,blaOXA-48-like, blaIMPlike,blaGES-like,blaGIM-like,blaAIM-like,blaBIC-like,blaDIM-like,blaIMI-like,blaNMC-Alike,blaSIM-like,blaSPM-like,blaLMB-like,blaFRI-like,blaSME-like,blaKHM-like,blaBKClike,blaOXA-23-like,blaOXA-51-like and blaOXA-58-like) and their variants were detected using ARIBA v2.6.1. [5].

\subsubsection{Identification of ESBL genes, $\mathrm{AmpC}$ genes and porin alterations.}

As with the carbapenemase genes, the presence of ESBL and AmpC genes was detected using ARIBA v.2.6.1. Porin genes ompK35 and ompK36 were also detected with ARIBA and checked for completeness. Genes were considered to be defective if the gene was found to be absent, fragmented or interrupted, or if frameshift or stop codon mutations were visible.

\subsubsection{Identification of non-ß-lactam resistance genes.}

Resistance genes for other antimicrobial families such as aminoglycosides, fluorquinolones, etc. were also searched using ARIBA v.2.6.1. [5].

\subsubsection{Identification of plasmid incompatibility groups and Annotation.}

Incompatibility groups were determined using PlasmidFinder function of ARIBA v.2.6.1., whereas annotation of the plasmids was performed using Prokka v.1 [6]. 


\subsection{Susceptibility Testing}

Antimicrobial susceptibility testing was performed by broth microdilution on the 234 isolates that were included in the genomic analysis using the automates system MicroScan WalkAway (Beckton Dickinson). The minimal inhibitory concentrations (MICs) of ampicillin, amoxicillin clavulanate, amikacin, cefepime, cefotaxime, cefoxitin, ceftazidime, cefuroxime, ciprofloxacin, colistin, ertapenem, fosfomycin, gentamicin, imipenem, norfloxacin, piperacillin-tazobactam, tigecycline, trimethoprim-sulfamethoxazole, meropenem and levofloxacin were determined. Antibiotics being tested varied depending on the origin of the sample. Susceptibility breakpoints were interpreted according to the recommendations of the Clinical and Laboratory Standards Institute (CLSI) [7].

\section{Results and Discussion}

\subsection{Isolates, Sequence-Types and Susceptibility Testing}

Genome sequences were obtained for 234 isolates obtained from both clinical and surveillance samples during a four-year period of time. All of them were non-susceptible or showed intermediate susceptibility to carbapenems.

Phylogenetic lineages in K.pneumoniae were determined according to their sequence type (ST). Among the 35 different STs identified, ST101, ST11 and ST307 were the most prevalent ones. The other STs found had less than 6 isolates each one. In figure 1 the sequence-type distribution is shown.

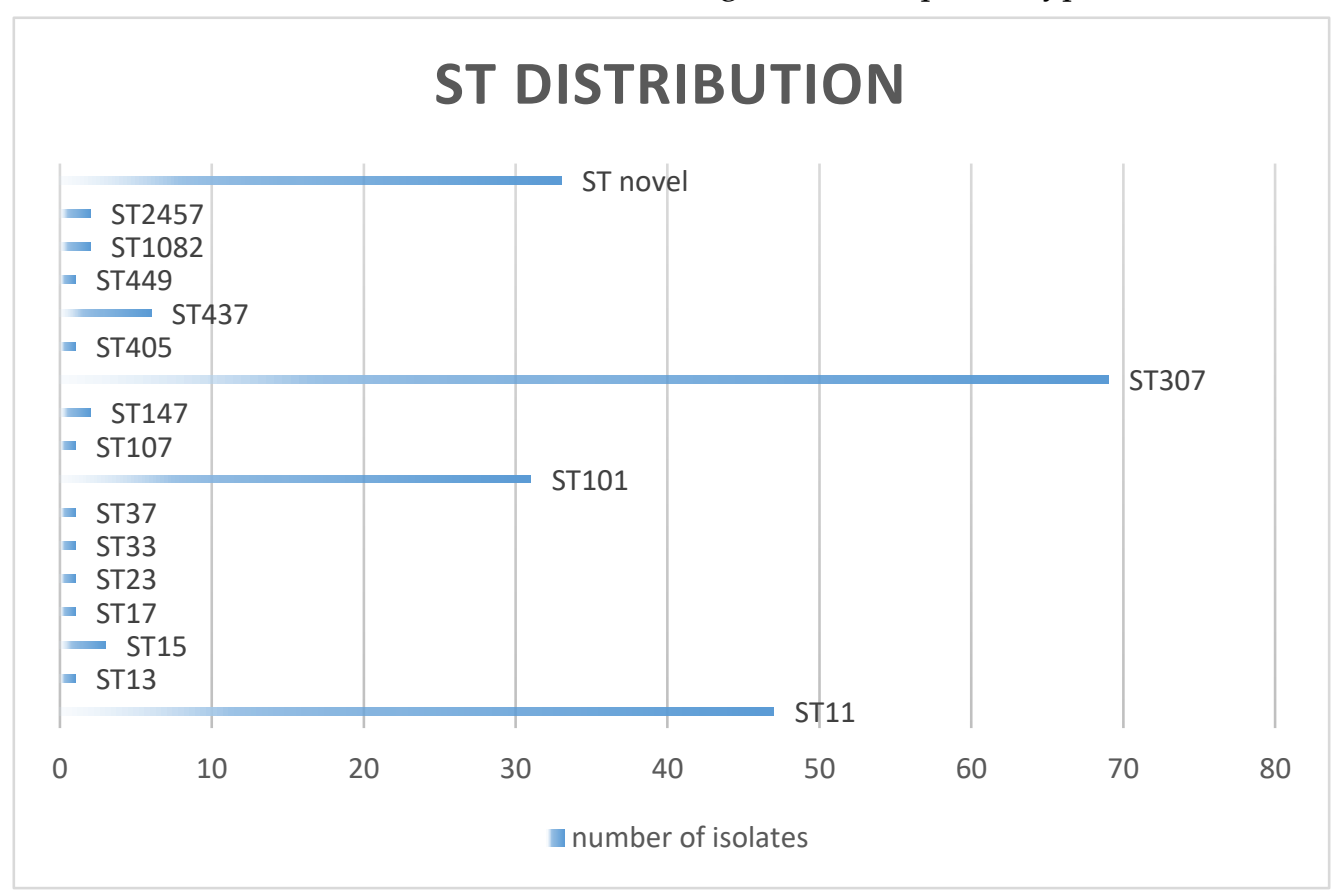

Figure 1. ST distribution of the $\mathrm{X}$ isolates included in the study.

Susceptibility testing of the 234 strains revealed that more than $95 \%$ were non-susceptible to third-generation cephalosporins (MICs> $4 \mathrm{mg} / \mathrm{L}$ ), and more than $90 \%$ were also non-susceptible to quinolones. Resistance to imipenem, meropenem, and ertapenem in all isolates was variable (MICs ranging from 1 to $>8 \mathrm{mg} / \mathrm{L}$ ). A moderate resistance, around $50 \%$, was shown to tigecycline (MICs $>2 \mathrm{mg} / \mathrm{L}$ ), gentamicin (MIC $>4 \mathrm{mg} / \mathrm{L}$ ) and fosfomycin (MICs $>32 \mathrm{mg} / \mathrm{L}$ ). Resistance of approximately $30 \%$ was present towards trimethoprim-sulfamethoxazole. Colistin turned out to be the one antibiotic with the highest susceptibility rates (MICs $<2 \mathrm{mg} / \mathrm{L}$ ).

Distribution of antimicrobial susceptibility of all 234 CRKP isolates is shown in Table 1. 
Table 1. Resistance rates against 20 antimicrobial agents assessed in 234 carbapenem-resistant Klebsiella pneumoniae isolates.

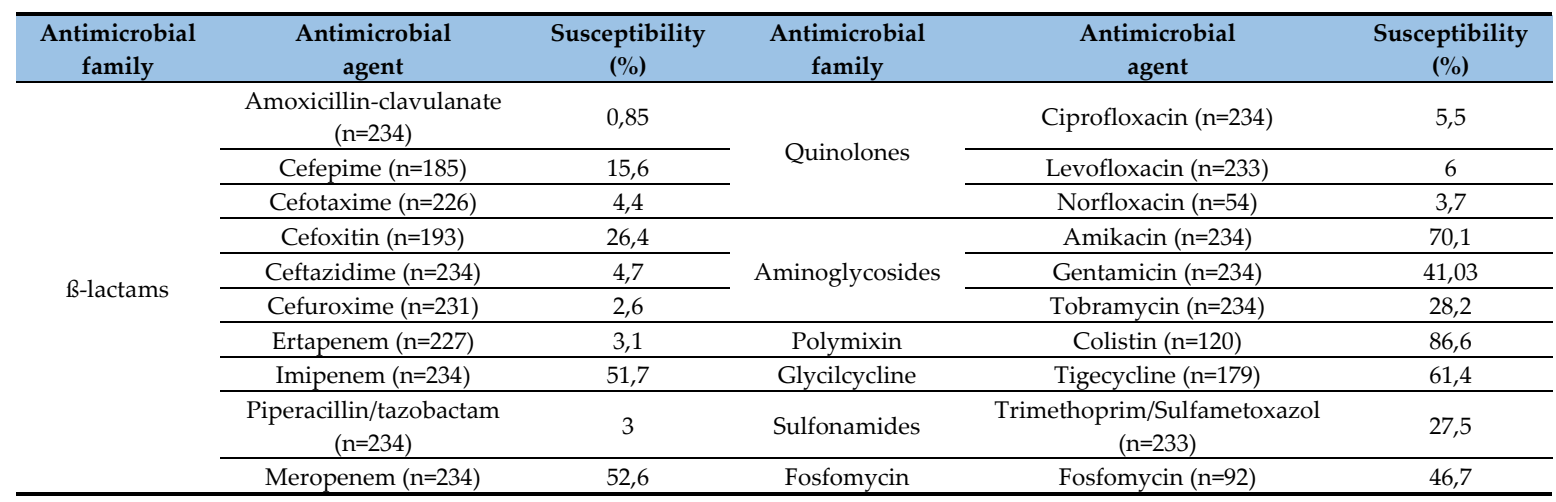

\subsection{Plasmid Profiles}

Several incompatibility groups were detected (see Figure 2), being the most frequent ones IncL/M harboring OXA-48 gene, IncR harboring resistance genes such as Qnr , arr3, armA and aac6'Ibcr and the IncF family (IncFIB and IncFII), harboring NDM-1 and other resistance genes such as CTX-M-15, TEM-1, and aminoglycosides and tetracyclines resistance genes.

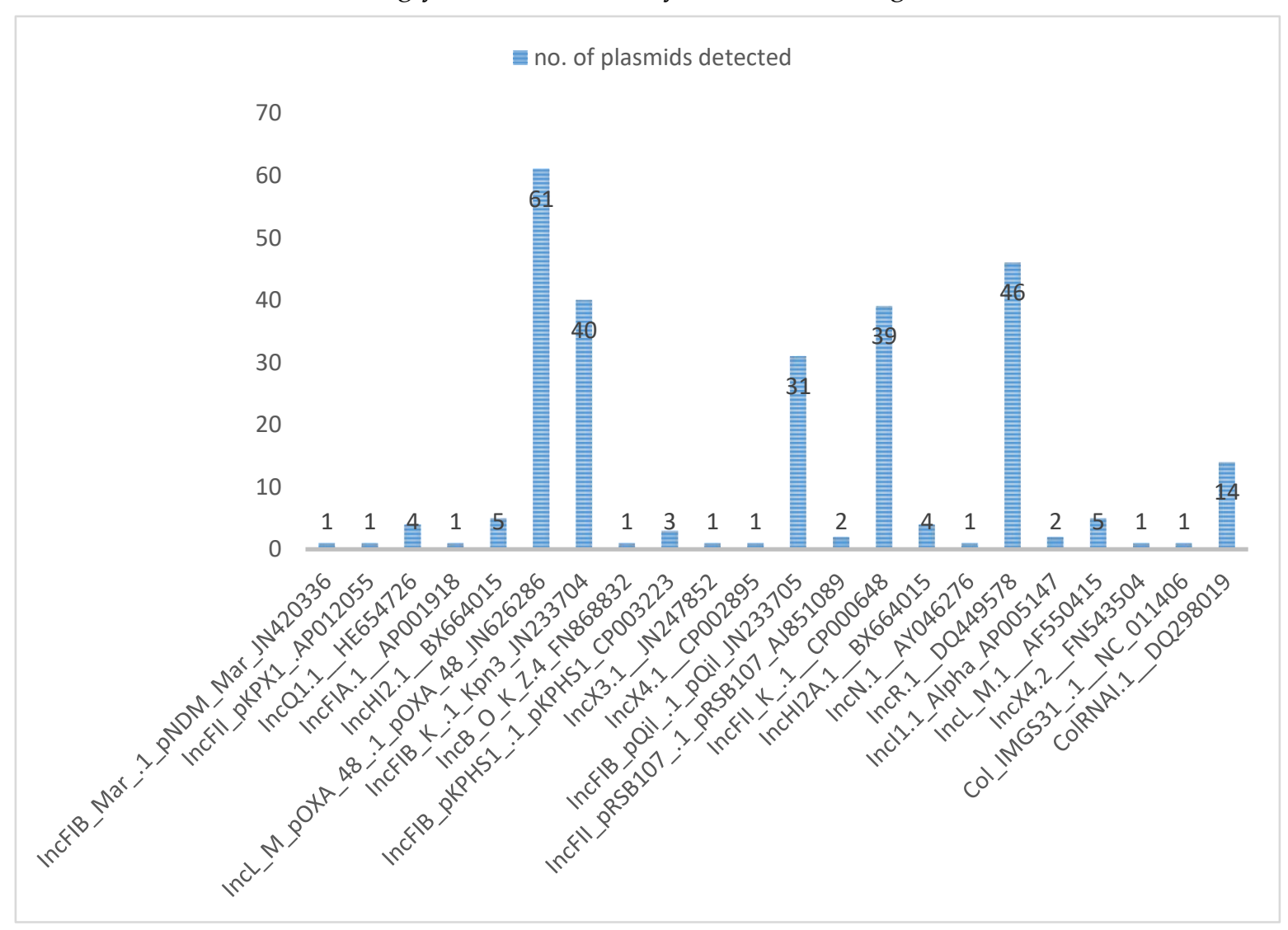

Figure 2. Plasmids detected using NGS.

\subsection{Resistance to $\beta$-lactams}

We searched the genomes for B-lactam resistance determinants that could be relevant to carbapenem resistance, and divided the entire collection into 2 " $\$$-lactams resistome groups". Group 1 contained one or more of any carbapenemase gene described in the literature before December 2018, regardless of other mechanisms; and group 2 contained any extended spectrum B-lactams gene 
and/or AmpC gene, without any obvious porin defects. It could not be possible to create a group considering porin defects, because the strains studied had no porin deficiency.

GROUP 1. One or more carbapenemases with or without other ß-lactams resistance genes.

Table 2. Genes identified.

\begin{tabular}{lccc}
\hline Gene(s) & Location & $\begin{array}{c}\text { Incompatibility } \\
\text { group }\end{array}$ & $\begin{array}{c}\text { Other B-lactams resistance determinants } \\
\text { located on the same plasmid }\end{array}$ \\
\hline OXA-48 & Plasmid & IncL/M & None \\
\hline \multirow{2}{*}{ NDM-1 } & Plasmid or & IncFIB/IncFII & TEM-1 \\
& Chromosome $(<1 \%)$ & DHA-1 \\
\hline
\end{tabular}

GROUP 2. B-lactamases other than carbapenemases, without any obvious porin defects and phenotypic resistance to carbapenems.

Table 3. Genes identified.

\begin{tabular}{ccc}
\hline Gene(s) & Location & Incompatibility group \\
\hline CTX-M group & Plasmid & IncFIB \\
\hline TEM-1 & Plasmid & IncFIB \\
\hline SHV & Plasmid/Chromosome & IncFIB \\
\hline DHA-1 & Plasmid & IncFIB \\
\hline OXA-1 & Plasmid & IncFIB \\
\hline
\end{tabular}

\subsection{Resistance to Quinolones}

Phenotypic resistance to quinolones was detected in more than $95 \%$ of the isolates. Several genes conferring resistance to these antimicrobials were found after whole-genome sequencing (see Table $4)$.

Table 4. Genes identified.

\begin{tabular}{ccc}
\hline Gene(s) & Location & Incompatibility group \\
\hline Aac6Ib-cr & Plasmid or chromosome & IncR \\
\hline Qnr & Plasmid & IncR \\
\hline Oqx & Chromosome & - \\
\hline
\end{tabular}

\subsection{Resistance to Aminoglycosides}

Table 5. Genes identified.

\begin{tabular}{ccc}
\hline Gene(s) & Location & Incompatibility group \\
\hline rmtF & Plasmid & IncFIB/IncFII \\
\hline armA & Plasmid & IncR \\
\hline Aac3 & Plasmid & IncFIB/IncFII \\
\hline Aad2 & Plasmid & IncFIB/IncFII \\
\hline Aph3 & Plasmid & IncFIB/IncFII \\
\hline Aph4 & Plasmid & IncFIB/IncFII \\
\hline
\end{tabular}

\subsection{Resistance to Fosfomycin and Tygecicline}

Fos $A$ providing resistance to fosfomycin was detected in $50 \%$ of the strains studied, being this resistance gene located in the chromosome. Regarding tygecicline, oqxA genes that may confer resistance were detected in all the strains studied.

\subsection{Resistance to Colistin}

Plasmid-borne mcr-1 resistance gene was not detected in the strains studied. 
Table 6. Phenotypic vs Genotypic resistance in 234 K.pneumoniae isolates.

\begin{tabular}{|c|c|c|c|}
\hline Antimicrobial & $\begin{array}{c}\text { Phenotypic } \\
\text { resistance } \\
(\%)\end{array}$ & Genes & $\begin{array}{c}\text { Level of agreement genotypic-phenotypic } \\
\text { expression (\%) }\end{array}$ \\
\hline Penicilins & 100 & $\begin{array}{c}\text { OXA-1 } \\
\text { CTX-M } \\
\text { SHV } \\
\text { TEM }\end{array}$ & 100 \\
\hline Cephalosporins & 90 & $\begin{array}{c}\text { CTX-M } \\
\text { SHV } \\
\text { TEM } \\
\end{array}$ & 100 \\
\hline Ertapenem & 97 & $\begin{array}{l}\text { OXA-48 } \\
\text { NDM-1 }\end{array}$ & 100 \\
\hline Aminoglycosides & 47 & $\begin{array}{c}\text { rmtF } \\
\text { armA } \\
\text { Aac3 } \\
\text { Aad2 } \\
\text { Aph3 } \\
\text { Aph4 }\end{array}$ & 63 \\
\hline Tetracyclines & 40 & TetR & 40 \\
\hline Fluorquinolones & 100 & $\begin{array}{c}\text { Aac6lb- } \\
\text { cr } \\
\text { Qnr } \\
\text { Oqx }\end{array}$ & 100 \\
\hline
\end{tabular}

Klebsiella pneumoniae plays a major role in the antibiotic resistance nowadays. The emergence of multiple resistances towards different antibiotic groups is frequently found in hospital-adapted $K$. pneumoniae isolates due to the accumulation of antimicrobial resistance genes that may be found on several plasmids forming what is known as "super resistome". Infections caused by these multidrug resistant microorganisms are associated with more adverse clinical outcomes and higher mortality rates [8]. In K.pneumoniae, carbapenemases are the main contributing factor to extensive drug resistance [1]. Therefore, understanding the genetic and mechanistic basis for multidrug resistance becomes crucial, as it may provide a hint on finding solutions to prevent its spread.

This study describes the resistome of carbapenem-resistant K.pneumoniae isolates recovered in Valencia (Spain), as well as the sequence types that are causing infections in our area.

In this work, with high troughoutput sequencing, we obtained the complete sequences of K.pneumoniae isolates.

After MLST typing, we found that the more frequently sequence types detected were ST101, ST11,ST307, ST147 and ST15 (these two less frequently detected), all of them considered high-risk clones of K.pneumoniae [9].These strains carried more than 50 resistance genes and showed phenotypic resistance to a variety of antibiotics, including B-lactams, aminoglycosides and quinolones, among others.

One particularly interesting finding in our work is that most of the antimicrobial resistance genes are encoded by three families of K.pneumoniae plasmids. For example, the resistance to aminoglycosides, tetracyclines and trimethoprim are not encoded by the chromosome, and completely depend on the presence of these three plasmids: IncL/M, IncF and IncR incompatibility groups. This is of major importance, as plasmids can easily be transferred between bacteria, disseminating this way antimicrobial resistance genes.

Oqx genes are relevant to efflux pumps, which means that antibiotics such as cephalosporins, carbapenems and quinolones are almost completely expelled from K.pneumoniae through its cell membrane. This would explain the multidrug resistance in the isolates of our study that were not harboring carbapenemase genes and yet turned out to be resistant to carbapenems and other antimicrobials.

Regarding agreement between phenotypic and genotypic detection of resistance, we did not find a $100 \%$ correspondence between both methods. In the case of tygecicline, for example, even though 
all the strains carried the oqxA which is believed to confer resistance to this antibiotic, less than $50 \%$ of the strains were resistant. The same happened with aminoglycosides, probably because the genes detected do not confer a high level of resistance to these antimicrobials and can be found to be phenotypically susceptible.

Another important finding in our study is the co-existence of genes coding for at least two classes of carbapenemase in K.pneumoniae, which has been reported worldwide [10], but co-harboring OXA48 and NDM-1 metallo-ß-lactamase is infrequently reported [11], with few reports worldwide and none in the area of the Valencian Community.

Clonality found in the strains studied is notorious, because this means that certain high-risk clones such as ST11, ST307 and ST101 continue to spread in hospital settings, transferring horizontally resistance genes to other K.pneumoniae sequence-types.

\section{Conclusions}

In conclusion, these super strains may be practically resistant to all available classes of antibiotics, posing a great challenge to clinicians due to limited available treatment options.

Whole-genome sequencing of carbapenem-resistant K.pneumoniae showed the presence of several plasmids, belonging mainly to IncF, IncR and IncL/M incompatibility groups. The genome encoded more than 50 drug resistance genes, of which more than $80 \%$ were plasmid-encoded.

Interestingly, all the strains belonging to a specific sequence-type were found to be harboring the same plasmids, and consequently very similar resistance genes, meaning that clonality is a characteristic of the isolates of K.pneumoniae in our area.

It is important to mention that a single strain of ST307 carbapenem-resistant K.pneumoniae harboring two classes of carbapenemase genes (blaoxA-48 and blanDM-1) has not previously been identified in other hospitals of our region. The strains of single-carbapenemase producing plus ESBLs genes, also described in our study, may already be far more prevalent than believed previously, especially regarding OXA-48 and CTX-M-1, which have already been widely reported in our country.

Application of new technologies such as whole-genome sequencing in clinical microbiology gives advantages when it comes to rapid therapy adjustment, consequently improving the patient's clinical outcomes.

Funding: This research did not receive any specific grant from funding agencies in the public, commercial, or non-profit sectors.

Acknowledgements: The authors want to thank all the team in the Microbiology Department at Valencia General Hospital.

Conflict of Interest: The authors have no competing interests to declare.

\section{References}

1. David, S., Reuter, S., Harris, S.R. et al. Epidemic of carbapenem-resistant Klebsiella pneumoniae in Europe is driven by nosocomial spread. Nat Microbiol 4, 1919-1929 (2019) doi:10.1038/s41564-019-0492-8

2. S. Navon-Venezia, K. Kondratyeva, A. Carattoli. Klebsiella pneumoniae: a major worldwide source and shuttle for antibiotic resistance

3. U.H. Trivedi, T. Cezard, S. Bridgett, A. Montazam, J. Nichols, M. Blaxter, K. Gharbi, Quality control of nextgeneration sequencing data without a reference. Front. Genet. 5, 111 (2014). doi: 10.3389/fgene.2014.00111

4. Diancourt, L., Passet, V., Verhoef, J., Grimont, P. A. \& Brisse, S. Multilocus sequence typing of Klebsiella pneumoniae nosocomial isolates. J. Clin. Microbiol. 43, 4178-4182 (2005).

5. Hunt, M. et al. ARIBA: rapid antimicrobial resistance genotyping directly from sequencing reads. Microb. Genom. 3, e000131 (2017).

6. Seemann T. (2014). Prokka: rapid prokaryotic genome annotation. Bioinformatics 30 2068-2069. 10.1093/bioinformatics/btu153

7. Clinical \& Laboratory Standards Institute: CLSI Guidelines [Internet]. [cited 2019 Sep 26]. Available from: https://clsi.org/

8. Nordmann P, Dortet L, Poirel L. Carbapenem resistance in Enterobacteriaceae: here is the storm! Trends Mol Med. 2012 May;18(5):263-72. 
9. Gonçalves, Guilherme \& Furlan, João Pedro \& Vespero, Eliana \& Pelisson, Marsileni \& Stehling, Eliana \& Pitondo-Silva, André. (2017). Spread of multidrug-resistant high-risk Klebsiella pneumoniae clones in a tertiary hospital from southern Brazil. Infection, Genetics and Evolution. 56. 10.1016/j.meegid.2017.10.011.

10. Lee CR, Lee JH, Park KS, Kim YB, Jeong BC, Lee SH. Global dissemination of carbapenemase-producing Klebsiella pneumoniae: Epidemiology, genetic context, treatment options, and detection methods. Vol. 7, Frontiers in Microbiology. Frontiers Media S.A.; 2016.

11. Codjoe F, Donkor E. Carbapenem Resistance: A Review. Medical Sciences. 2017 Dec 21;6(1):1. 